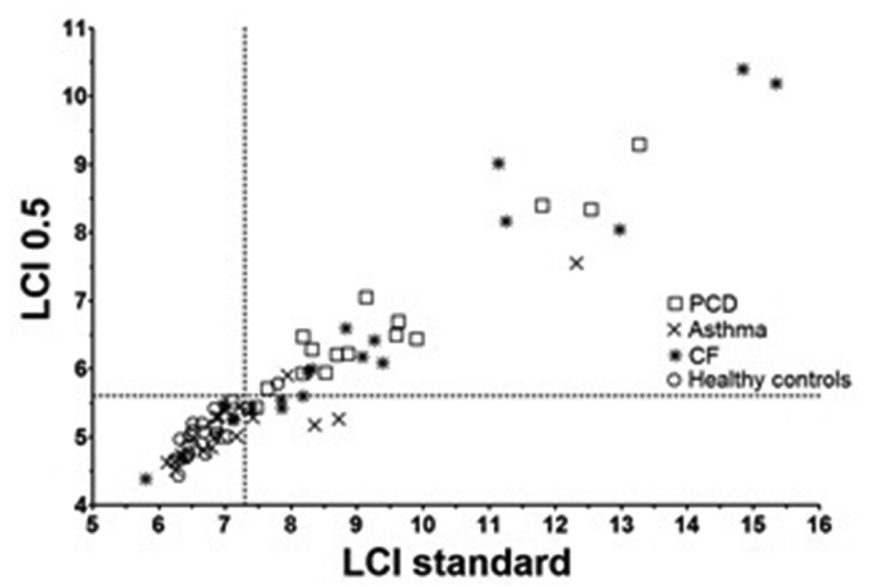

Abstract P205 Figure 1 Correlation between LCI0.5 and LClstd. Shortened MBW, LCI0.5, correlated significantly with LClstd with $r$ values of $0.84,0.96$ and 0.92 in asthma, CF and PCD groups respectively. The dotted lines indicate the upper limits of normal: LCI0.5 is 5.6 and $\mathrm{LClstd}$ is 7.3

with LCIstd and change following an intervention were considered.

\section{P206 CHANGES IN INDICES DERIVED FROM MULTIBREATH WASHOUT (MBW) FOLLOWING TREATMENT WITH IVACAFTOR IN PATIENTS WITH CYSTIC FIBROSIS}

KML Harman, SJ Irving, K Bayfield, C Saunders, EJ Spearing, JC Davies. Imperial College, London, UK

\subsection{6/thoraxjnl-2014-206260.335}

Background Lung clearance index (LCI) is a measure of gas mixing inhomogeneity derived from multi-breath wash (MBW) out techniques, which has been shown to be more sensitive than conventional spirometry/ $\mathrm{FEV}_{1}$. The potentiator, Ivacaftor, led to improvement in LCI in patients with mild cystic fibrosis (CF) lung disease however its utility as an outcome measure in more severe disease requires further investigation. Whilst LCI reflects overall ventilation heterogeneity, analysis of the phase III slopes of successive breaths in the MBW; known as Sacin and Scond; are thought to reflect the ventilation heterogeneity generated at branch points in the acinar and conductive lung zones respectively. This study aimed to explore changes in the indices derived from analysis of MBW following a year of treatment with Ivacaftor.

Method A prospective study was performed between March 2013 and April 2014 on patients with the G551D mutation and eligible for clinically prescribed Ivacaftor. MBW (Innocor SF6 technique) and spirometry were performed immediately prior to commencing Ivacaftor, and at a clinic visit following 9-12 months therapy. $\mathrm{FEV}_{1}$ is calculated using Stanojevic references and all data are expressed as mean (SD). Paired data were analysed with a Wilcoxon rank sum test and correlations with Spearman's rank correlation. The null hypothesis was rejected at $\mathrm{p}<0.05$.

Results 8 patients were enrolled with ages ranging from 6-27 years. $\mathrm{FEV}_{1}$ increased from $68.7(17.2) \%$ before treatment to $80.1(16.3) \%$ after 9-12 months ( $\mathrm{p} 1$ and LCI did not correlate with each other.

Discussion Patients prescribed Ivacaftor demonstrated improvements in both conventional $\mathrm{FEV}_{1}$ and the newer measure of
LCI; improvement was not limited to patients with milder disease and was seen throughout the group. In this study, the phase III slope measures did not appear to add further value to the LCI. It is possible that this reflects under powering in this small group; further data will be obtained.

\section{P207 RELIABILITY OF MEASUREMENTS USING INNOCOR BREATH BY BREATH ANALYSER DURING A MAXIMAL EXERCISE TEST IN CYSTIC FIBROSIS PATIENTS}

${ }^{1} \mathrm{KJ}$ Bayfield, ${ }^{2} \mathrm{M}$ McGovern, ${ }^{1} \mathrm{AJ}$ Simpson, ${ }^{2} \mathrm{M}$ Embley, ${ }^{2} \mathrm{~S}$ Cunningham, ${ }^{1} \mathrm{JC}$ Davies, ${ }^{1} \mathrm{EWFW}$ Alton, ${ }^{2} \mathrm{JA}$ Innes. ${ }^{1}$ Imperial College London, London, UK; ${ }^{2}$ University of Edinburgh, Edinburgh, UK

\subsection{6/thoraxjnl-2014-206260.336}

Introduction Cardiopulmonary exercising testing (CPET) is considered the gold standard to study exercise capacity as an endpoint in clinical trials. Originally the UKCFGTC used the shuttle walk test for exercise capacity measurement but this proved inappropriate for mild, fit cystic fibrosis (CF) patients in our trial cohort ( $\mathrm{FEV}_{1}$ 50-90\% predicted). The Innocor device uses photoacoustic gas detection technology and offers metabolic measurement but has not previously been validated for CPET in CF. Aim To compare the Innocor with known reliable CPET machines to see if it is suitable to take forward into a multi dose clinical trial of gene therapy.

Methods 12 CF patients (7 Male, 14-47 years) participated in the study recruited from London and Edinburgh sites. They performed two incremental cycle ergometer exercise tests to exhaustion (adapted Godfrey protocol) with breath by breath analysis assessed using a reference system (Jaeger Masterscreen PFT, London; Pulmolink Medisoft, Edinburgh) or the Innocor device. All tests were randomly ordered, completed at least $24 \mathrm{~h}$ apart, with no more than two week's separation.

Results $\mathrm{VO}_{2} \max$ and $\mathrm{V}_{\mathrm{E}}$ max were comparable between the Innocor and reference systems $(p=0.1790$ and $p=0.7642$ respectively; paired t tests). For VO2max, Bland Altman analysis showed the mean difference [Reference equipment-Innocor] was $-0.026 \mathrm{l} / \mathrm{min}$ and the $95 \%$ confidence interval was -0.27 to 0.22 1/min (see Figure). In our experience the Innocor heart rate (HR)

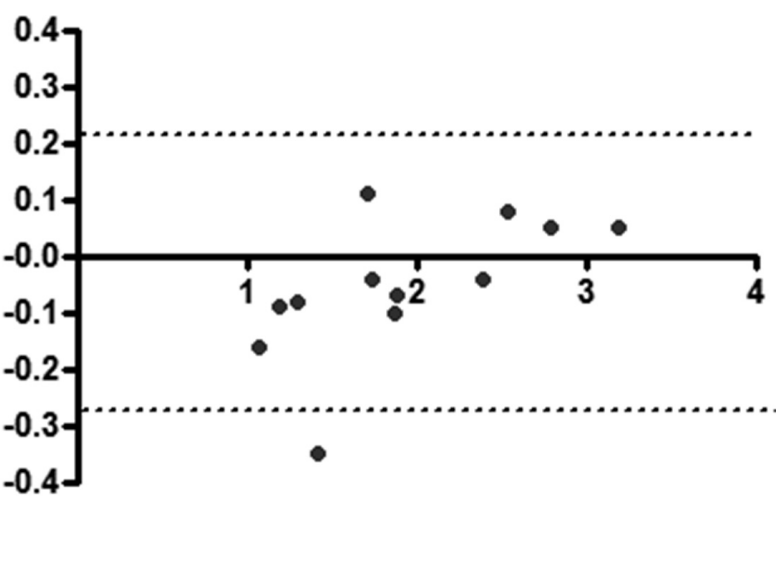

\section{Mean VO2max (I/min)}

Abstract P207 Figure 1 Bland Altman plot: the difference between $\mathrm{VO}_{2}$ max measured by Innocor vs Reference methods. 